\title{
Sequencial luminoso controlado por Bluetooth
}

\author{
Luminous sequencial controlled by Bluetooth
}

\author{
Amanda Wohlfahrt Reis ${ }^{1}$, Odair José Iaronk ${ }^{2}$ e Celso Becker Tischer ${ }^{3}$ \\ ${ }^{1}$ Universidade Federal de Santa Maria, Cachoeira do Sul, Brasil \\ amanda_wreis@hotmail.com \\ ${ }^{2}$ Universidade Federal de Santa Maria, Cachoeira do Sul, Brasil \\ odair.iaronka@gmail.com \\ ${ }^{3}$ Universidade Federal de Santa Maria, Cachoeira do Sul, Brasil \\ cbtischer@gmail.com
}

\begin{abstract}
Resumo
A utilização de microcontroladores teve crescimento nos últimos anos no desenvolvimento de sistemas eletrônicos devido a suas pequenas dimensões e capacidade de controlar processos. A aplicação de microcontroladores é significativa em diversos setores, tais como: industrias, residencial, entretenimento, telecomunicações, automotivas, transporte e aviação. O arduino é uma plataforma eletrônica de baixo custo, open source, de prototipagem projetada com um microcontrolador embutido baseado em hardware e software de fácil utilização, que pode ser programada a fim de configurar as entradas e saidas entre os componentes externos conectadas a plataforma. Assim, visando a transformação do entretenimento em uma forma de aprendizagem, este trabalho apresenta o desenvolvimento de um sequencial luminoso baseado em arduino programado em linguagem C. O sistema é composto por 25 LED's distribuidos de forma estratégica para a visualização das sequencias pré-programadas. No projeto utilizou-se o arduino MEGA 2560 e um módulo Bluetooth para comunicação sem fio entre o microcontrolador e dispositivos multifuncionais como smartphone's, um aplicativo desenvolvido no MIT APP INVENTOR controla o sistema, proporcionando ao usuário a capacidade de escolha da rotina além da velocidade e intensidade luminosa do conjunto.
\end{abstract}

Palavras-chave: Arduino; Bluetooth; LED

\section{Abstract}

The use of microcontrollers has been growing in the last years, in the development of electronic systems, due to their small dimensions and ability to control process. The application of microcontrollers is significant in several sectors, such as: industrial, domestic, entertainment, telecommunication, automotive, transport and aviation. Arduino is an electronic platform low-cost, open source, prototyping designed whit a built-in microcontroller based on easy-to-use hardware and software that can be programed to configure the inputs and outputs between the external components connected to the platform. Therefore, aiming the transformation of entertainment into a form of learning, this work presents the development of a luminous sequential programmed in C language. The system is composed by 25 LED's strategically distributed for the visualization of the preprogrammed sequences. In the project the MEGA 2560 arduino was used and a Bluetooth module for wireless communication between the microcontroller and multifunction devices like smartphone's, an application develop in MIT APP INVENTOR controls the systems, giving the user the ability to choose the routine besides the speed and luminous intensity of the set.

Keywords: Arduino; Bluetooth; LED 


\section{Introdução}

O Arduino foi originalmente projetado como um recurso para auxiliar os estudantes no ensino de eletrônica, sendo que em 2005 ele foi comercialmente lançado por Massimo Banzi e David Cuartielles, tornando-se um produto de sucesso entre fabricantes e estudantes devido a sua fácil utilização e a durabilidade no qual proporciona (MONK, 2013). O Arduino é uma plataforma projetada através de um microcontrolador de programação específica dotado de pinos de entrada e saída de energia e sinal que pode ser facilmente aplicada nos mais diversificados projetos eletrônicos e elétricos.

Fundamentalmente os Arduinos são desenvolvidos de maneira semelhante, variando somente em algumas de suas características como número de portas, conexões, tamanho e sistemas adicionais. Na Figura 1 pode-se visualizar o esquema de conexões do modelo de Arduino Mega 2560, o mesmo possui 54 pinos de entradas/saídas digitais, das quais 15 podem ser do tipo Pulse Width Modulation (PWM), também possui 16 entradas analógicas, além de pinos de alimentação com duas tensões padrão $(3,3 \mathrm{~V}$ e $5 \mathrm{~V})$, além dos demais pinos de configuração e alimentação.

Figura 1 - Visão geral da placa de Arduino Mega 2560

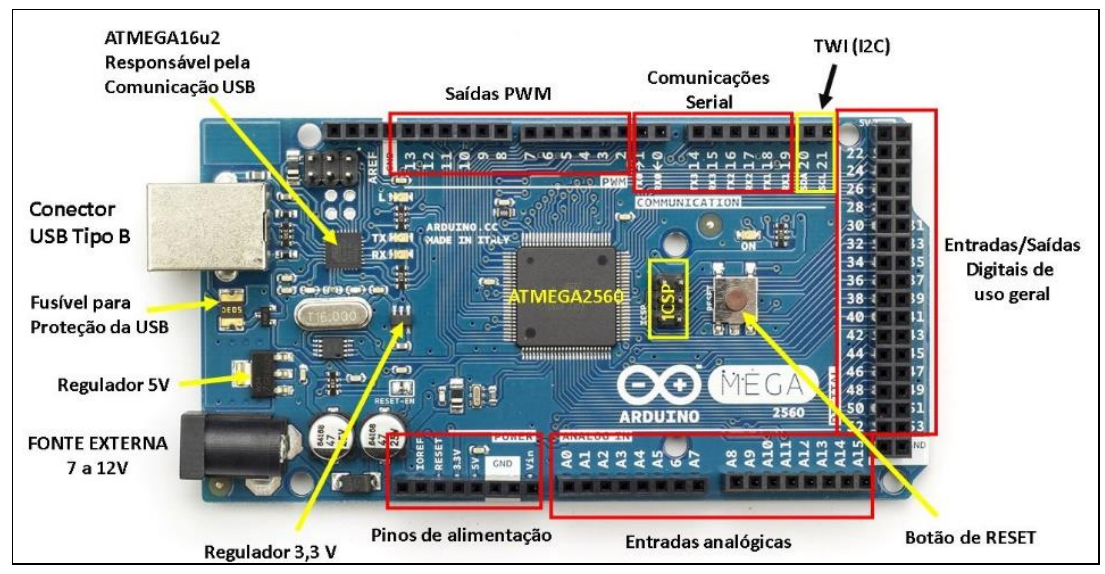

A linguagem de programação utilizada para realizar a programação deste Arduino, é a linguagem C. Segundo Schildt (1997), a linguagem C é classificada como uma linguagem de médio nível porque combina elementos de linguagens de alto nível com funcionalidades da linguagem Assembly, linguagem de mais baixo nível, com comunicação direta com o hardware.

A linguagem $\mathrm{C}$ foi criada para que o programador possa desenvolver códigos estruturados e modulares, tendo como resultado mais legibilidade e documentação, além de ter a vantagem de serem bastante compactos e de execução rápida. Programas em C podem ser desenvolvidas em várias partes separadas por diferentes usuários e depois unidas para formar um único programa, isto significa que podem ser criadas bibliotecas de funções para serem distribuídas e usadas sem que necessariamente seja conhecido o código fonte de cada uma delas (MIZRAHI, 2008).

Considerando a grande aplicabilidade dos doidos emissores de luz, Light Emitting Diode (LED), aplicados em anúncios, vitrines, salões de festas e em muitos outros, aliada a programação facilitada em Arduino, o presente artigo apresenta o desenvolvimento de um sequencial luminoso, utilizando 25 LED’s. O projeto contém dez rotinas distintas com velocidade de execução e intensidade luminosa ajustável pelo usuário via Smartphone. A programação do Arduino foi realizada em 
linguagem C, simulado no software Proteus e implementado no Arduino MEGA 2560, e além disso, foi desenvolvido um aplicativo para que o usuário interaja via Bluetooth com os comandos do projeto.

\section{Metodologia}

A partir da proposta de desenvolver um sequencial luminoso com mais recurso do que os utilizados atualmente, o primeiro passo do projeto se baseou em criar as diferentes rotinas que os LED's seguiriam, essas rotinas foram criadas de forma aleatória de acordo com a imaginação do projetista, um exemplo básico é a sequência que imita o ponteiro dos segundos de um relógio analógico. Após a definição das rotinas a serem implementadas neste projeto, deu-se início a etapa de programação. Para essa etapa utilizou-se o software do Arduino disponibilizado junto ao hardware utilizado. A Figura 2 apresenta o fluxograma utilizado para a programação desenvolvida.

Figura 2 - Fluxograma da programação

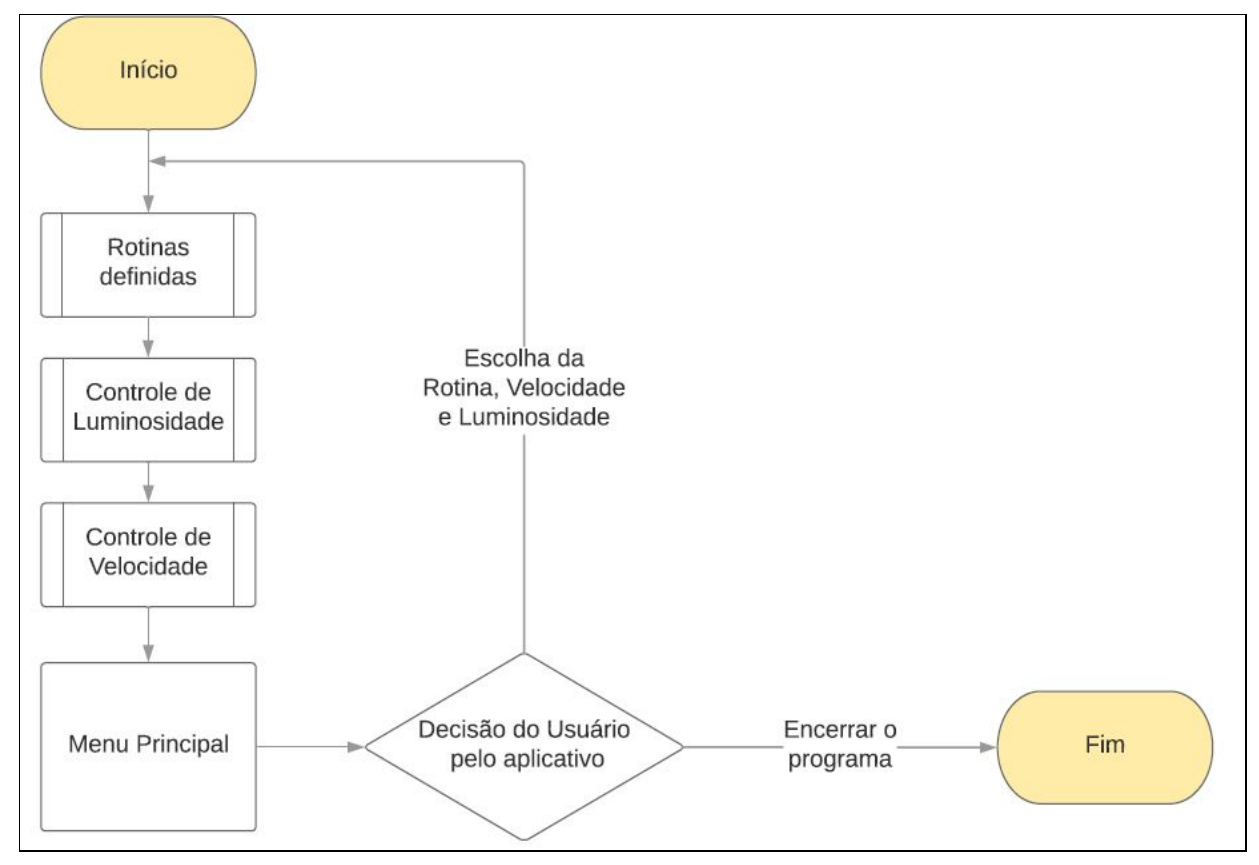

A linguagem $\mathrm{C}$ se torna essencial nessa fase, cada rotina teve uma programação separada dentro do código, assim uma vez com o código rodando cada rotina era compilada separadamente para realizar suas funções especificas. Após a realização dos dez códigos responsáveis pelas rotinas, utilizou-se do software Proteus para efetuar a simulação. Para a melhor visualização dos efeitos reproduzidos pelos LED’s a posição de cada um deles foi estratégica, o esquema utilizado para visualização e simulação do circuito pode ser visto na Figura 3.

Para a simulação do conjunto foi utilizado o Arduino MEGA 2560, o que é ideal para projetos que controlem grandes quantidades de LED's, devido ao seu grande número de entradas e saídas. Sua placa contém 54 entradas e saídas digitais, 14 delas são saídas PWM analógicas, e também conta com 16 pinos de entrada analógica.

Com o intuito de ter uma comunicação sem fio entre o Arduino e o dispositivo multifuncional optou-se pela comunicação Bluetooth, a qual é uma tecnologia desenvolvida pela Ericsson utilizada para comunicação sem fio de campo 
próximo, ou seja, seu alcance e velocidade são limitados, porém, fornece segurança e resolução na transferência de dados. O dispositivo multifuncional a ser utilizado para essa comunicação será um Smartphone com conexão Bluetooth.

Figura 3 - Simulação no software Proteus

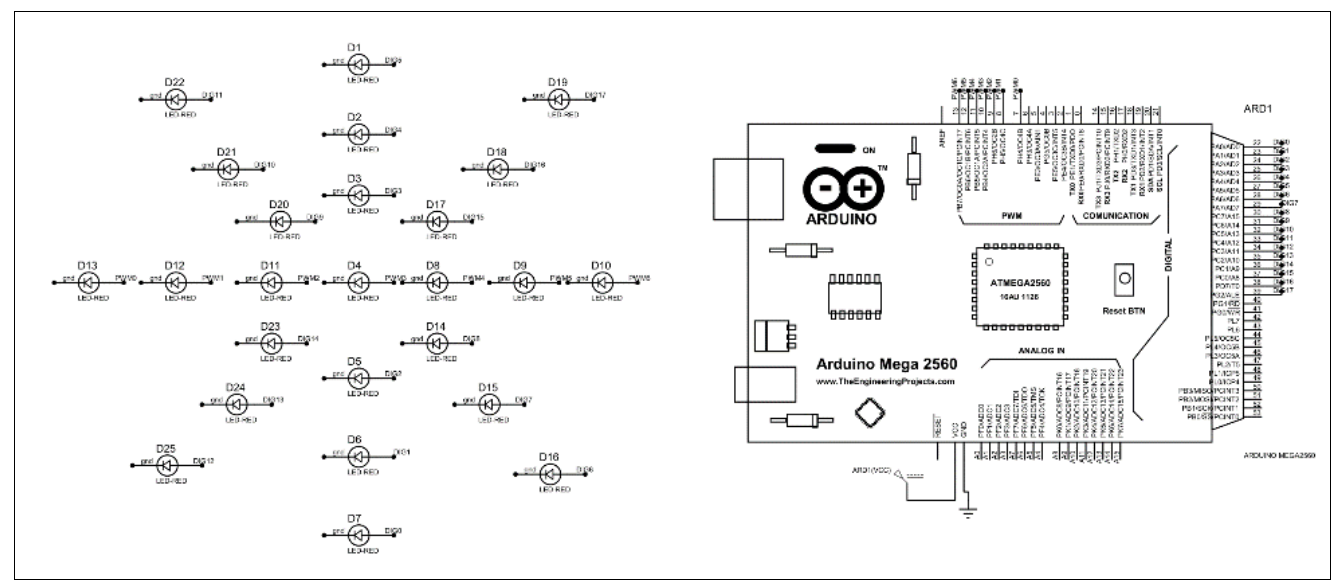

Desta forma, dispondo do site MIT APP INVENTOR foi desenvolvido um aplicativo para viabilizar a comunicação entre o dispositivo multifuncional e o Arduino. O aplicativo criado conta com diversas opções de funcionamento, sendo a conexão com o módulo Bluetooth a primeira a ser realizada. No aplicativo todas as rotinas dos LED’s são demonstradas, no qual, o usuário tem a possibilidade de escolher a rotina desejada, a possibilidade de controlar a intensidade luminosa e velocidade dos LED's. Além disso, o aplicativo oferece um botão “stop” com a opção de parar o processo a qualquer momento. Para uma melhor visualização, optou-se por adicionar um display ao conjunto que apresenta o número da rotina que foi escolhida, além da velocidade dos LED’s e a porcentagem de sua intensidade luminosa. Um esquemático entre as rotinas, display, comunicação sem fio e o aplicativo desenvolvido é apresentado na Figura 4.

Figura 4 - Fluxograma do funcionamento do projeto

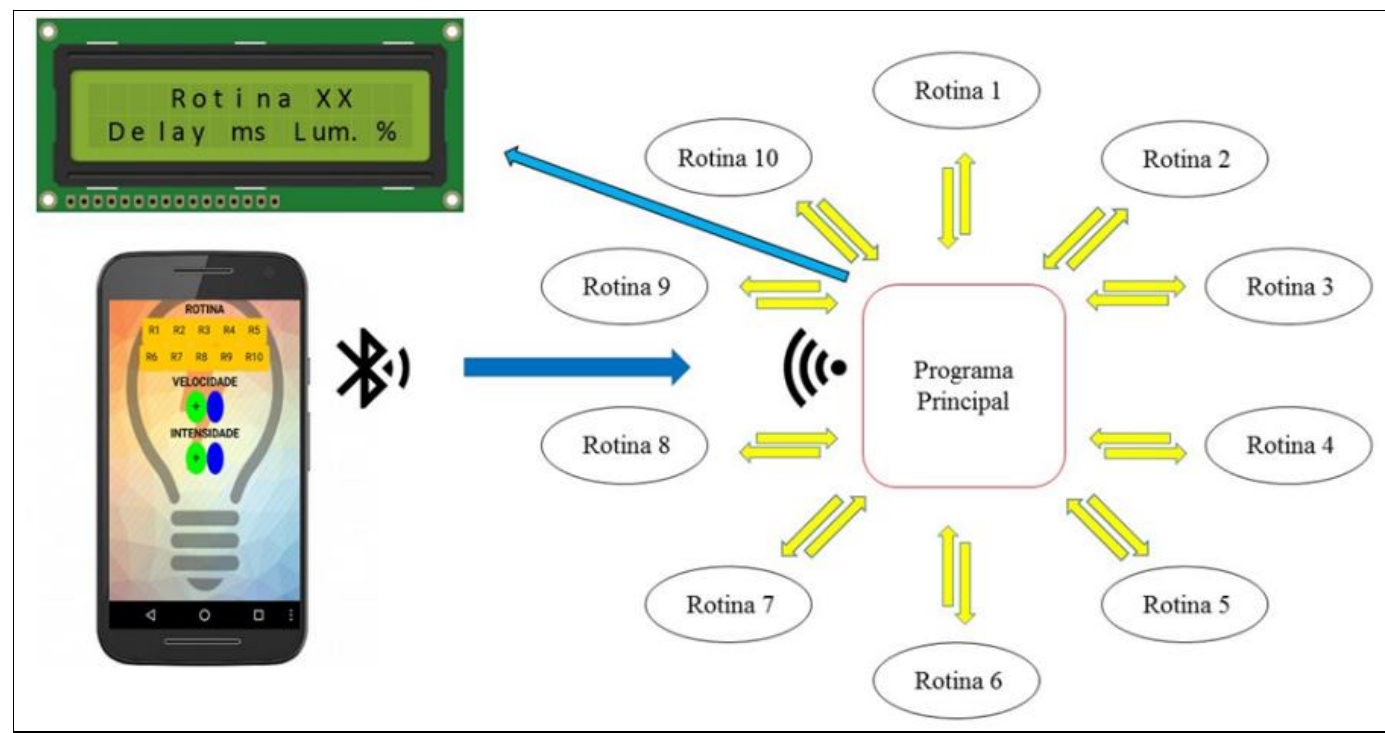




\section{Resultados}

Após a realização das simulações necessárias e das demais etapas apresentadas na metodologia, a primeira montagem prática se fez junto ao laboratório de Engenharia Elétrica da Universidade Federal de Santa Maria (UFSM) - Campus Cachoeira do Sul, com a utilização de uma Protoboard. A partir da verificação do correto funcionamento do protótipo, a confecção de um protótipo foi realizada. Para isto, utilizou-se uma caixa de PVC (Polyvinyl chloride) e de uma placa de circuito impresso para alocação dos LED’s, confeccionada em laboratório com demais periféricos. A primeira implementação prática do projeto utilizando a Protoboard pode ser observada na Figura 5.

Figura 5 - Implementação prática em Protoboard

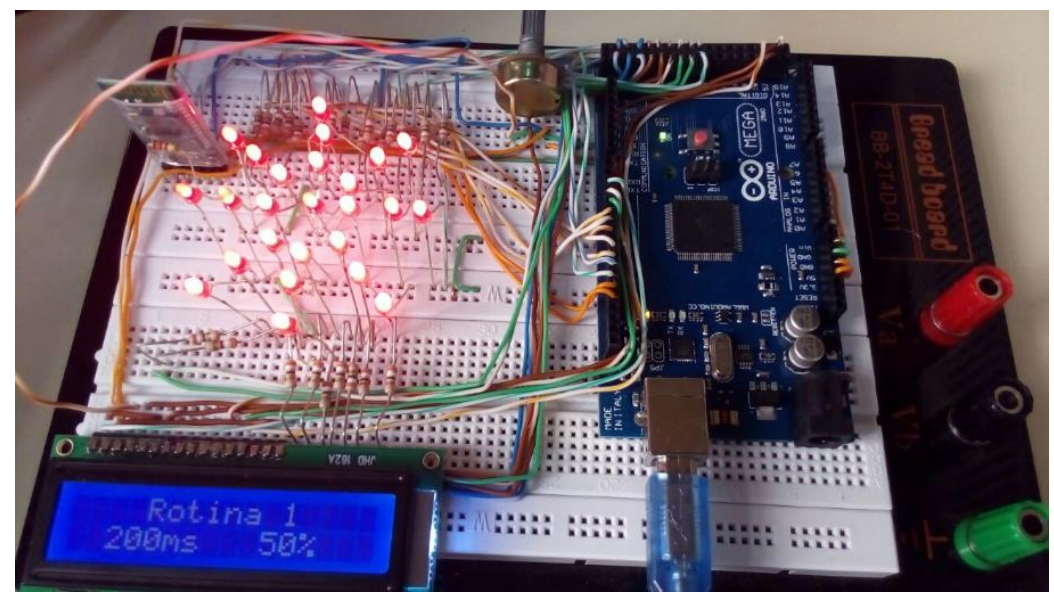

De modo que na primeira implementação prática obteve-se os resultados esperados, foi realizada uma segunda implementação prática, na qual foi desenvolvido um protótipo móvel, em virtude de que o mesmo oferece um aspecto favorável para apresentação ao público. O resultado final do protótipo pode ser visualizado na Figura 6.

Figura 6 - Implementação em laboratório. (a) Foto interna do protótipo (b) Foto externa do protótipo

(a)

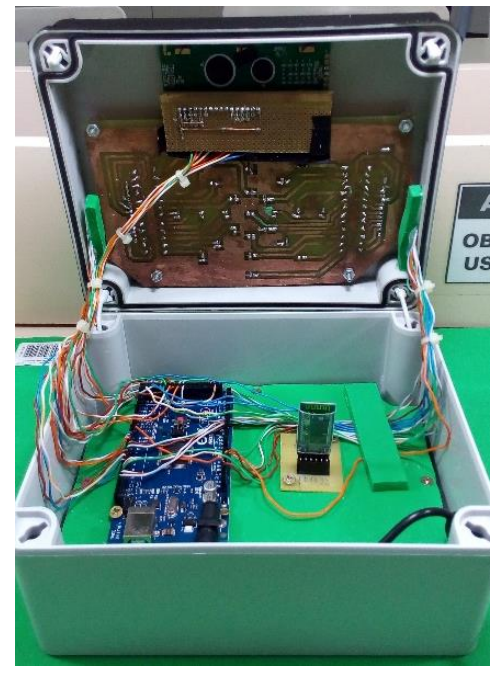

(b)

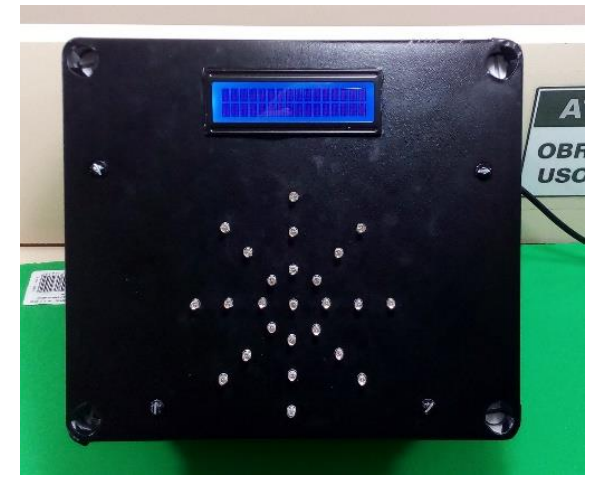


Para a comunicação sem fio com o conjunto associado ao Arduino um aplicativo foi criado, conforme mencionado anteriormente. O aplicativo conta com o botão para realizar a conexão Bluetooth, um botão para cada rotina desenvolvida, sendo o botão R10 estabelecido como sequência aleatória. O aplicativo conta também com os botões para aumentar e diminuir a velocidade das sequências assim como a intensidade luminosa dos LED's, além disso conta com o botão "Stop" que ao pressiona-lo interrompe a sequência e apaga todos os LED's, neste momento é apresentada uma mensagem de "Desligado" no display do conjunto. A interface gráfica do aplicativo criado é apresentado na Figura 7.

Figura 7 - Interface gráfica do aplicativo desenvolvido.

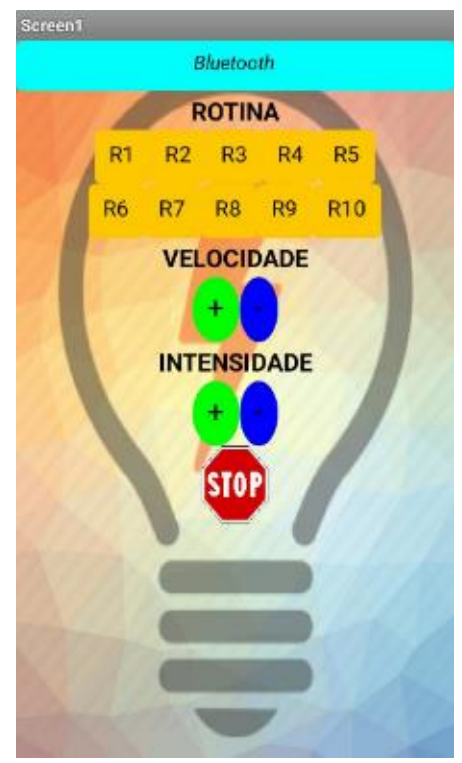

\section{Conclusões}

Com o desenvolvimento deste sequencial luminoso foi possível aperfeiçoar o conhecimento sobre a utilização do Arduino, da aplicabilidade do módulo Bluetooth para comunicação sem fio, além do desenvolvimento de aplicativos para smartphones o que resulta em um diferencial para o projeto. Sendo assim, esse conceito pode ser comparado com o princípio de automação residencial, o qual se baseia no controle de diversas funções no interior de uma residência, trazendo conforto e praticidade para os usuários.

\section{Agradecimentos}

Agradecimento especial ao Professor Celso Becker Tischer pelo auxílio e apoio no desenvolvimento do trabalho e também a Universidade Federal de Santa Maria, campus Cachoeira do Sul pela disponibilidade dos materiais utilizados na implementação prática do projeto. 


\section{Referências}

EVANS, M.; NOBLE, J; HOCHENBAUM, J. Arduino em Ação. São Paulo. Novatec. 2013.

MCROBERTS, M. “Arduino Básico”. São Paulo. Novatec. 2011.

MONK, S. Programação com Arduino >>começando com sketches, Bookman Editora LTDA. Porto Alegre, RS. 2013.

MIZRAHI, V. V. Treinamento em Linguagem C. São Paulo: Pearson Prentice Hall, 2008.

SARTORI, G., MOLINA, L. A., LIMA, W. C. G. Desenvolvimento de um sistema microcontrolado de baixo custo utilizando smartphone para aplicações de automação residencial. Trabalho de conclusão de curso, Departamento Acadêmico de Eletrônica. UTFP. 2015.

SCHILDT, H. C, Completo e Total. 3 ed. São Paulo: Pearson Makron Books, 1997.

BLOG USANAINFO [Internet], O que é arduino? De onde vem? Do que se alimenta? Available from: http://blog. usinainfo.com.br/o-que-e-arduino-de-onde-vem-do-que-se-alimenta. 